\title{
Synthesis and Characterization of Bi203 NPS and Photocatalytic Application with Methylene Blue
}

\section{Fowziya Shaik Ali S.A}

Department of Chemistry, Khadir Mohideen College, Adirampattinam. 614701(Affiliated to Bharathidasan University, Tiruchirappalli) India

\section{Faisal Al Marzouqi}

Department of Process Engineering, International Maritime College Oman, P.O. Box: 532, PC: 332, Falaj Al Qabail, Suhar, Oman

\section{Ragamathunnisa M}

Department of Physics, Government Arts College for Women (Auto), Pudukkottai, 622001, India Ismail Fathima M

Department of Physics, Arul Anandar College (Autonomus), Karumathur, Madurai. 625514

Mohamed Jahangir A. R.

Biyaq Oilfield Services LLC Post Box: 795, Mina Al Fahal PC, 116, Muscat, Sultanate of Oman Ayeshamariam A ( $\square$ ayeshamariamkmc@gmail.com)

Department of Physics, Khadir Mohideen College, Adirampattinam. 614701(Affiliated to Bharathidasan University, Tiruchirappalli) India

\section{Kaviyarasu K}

UNESCO-UNISA Africa Chair in Nanosciences/Nanotechnology Laboratories, College of Graduate Studies, University of South Africa (UNISA), Muckleneuk Ridge, P O Box 392, Pretoria,

\section{Short Report}

Keywords: Bismuth oxide, Nanoparticles, Photocatalysis

Posted Date: June 9th, 2021

DOI: https://doi.org/10.21203/rs.3.rs-577935/v1

License: (c) (1) This work is licensed under a Creative Commons Attribution 4.0 International License. Read Full License 


\section{Synthesis and characterization of $\mathrm{Bi}_{2} \mathrm{O}_{3}$ NPS and photocatalytic application with}

\section{methylene blue}

Fowziya Shaik Ali ${ }^{1}$, Faisal Al Marzouqi ${ }^{2}$, M. Ragamathunnisa ${ }^{3}$, M. Ismail Fathima ${ }^{4}$, A. R. Mohamed Jahangir ${ }^{5}$, A. Ayeshamariam ${ }^{6}$ and K.Kaviyarasu ${ }^{7,8}$

${ }^{1}$ Department of Chemistry, Khadir Mohideen College, Adirampattinam. 614701(Affiliated to Bharathidasan University, Tiruchirappalli) India

${ }^{2}$ Department of Process Engineering, International Maritime College Oman, P.O. Box: 532, PC: 332, Falaj Al Qabail, Suhar, Oman

${ }^{3}$ Department of Physics, Government Arts College for Women (Auto), Pudukkottai, 622001, India

${ }^{4}$ Department of Physics, Arul Anandar College (Autonomus), Karumathur, Madurai. 625514 ${ }^{5}$ Biyaq Oilfield Services LLC Post Box: 795, Mina Al Fahal PC, 116, Muscat, Sultanate of Oman

${ }^{6}$ Department of Physics, Khadir Mohideen College, Adirampattinam. 614701(Affiliated to Bharathidasan University, Tiruchirappalli) India

${ }^{7}$ UNESCO-UNISA Africa Chair in Nanosciences/Nanotechnology Laboratories, College of Graduate Studies, University of South Africa (UNISA), Muckleneuk Ridge, P O Box 392, Pretoria, South Africa.

${ }^{8}$ Nanosciences African network (NANOAFNET), Materials Research Group (MRG), iThemba LABS-National Research Foundation (NRF), 1 Old Faure Road, 7129, P O Box 722, Somerset West, Western Cape Province, South Africa.

Corresponding Email; ayeshamariamkmc@gmail.com 


\begin{abstract}
Nanoparticles of bismuth oxide were successfully synthesized by hydrothermal process which included Bismuth (III) nitrate $\left(\mathrm{Bi}\left(\mathrm{NO}_{3}\right)_{3} \cdot 6 \mathrm{H}_{2} \mathrm{O}\right)$, sodium hydroxide $(\mathrm{NaOH})$ and Nitric acid(HNO3) without further purification along with ultra-pure water. To investigate the structural, optical, and photocatalytic activity of two samples (1:5) and (1:6) respectively with two different $\mathrm{NaOH}$ precipitating agent molar ratios. The prepared nanoparticles were characterized by X-ray diffraction (XRD), UV-Vis's spectrometer, scanning electron microscope (SEM), Energy-dispersive X-ray spectroscopy (EDAX), Fourier transform infrared spectroscopy (FTIR) and X-ray Photo electron Spectroscopy (XPS). Based on the obtained results the hydrothermally synthesized Bi2O3 NPs exhibit good efficiency to photocatalytic degradation of Methylene blue under the irradiation of LED white light.
\end{abstract}

\title{
Keywords; Bismuth oxide, Nanoparticles, Photocatalysis.
}

\section{Introduction:}

Bismuth oxide $\left(\mathrm{Bi}_{2} \mathrm{O}_{3}\right)$ nano materials have a range of attractive properties including a high band gap (2-3.96eV), a high refractive index and photoluminescence and this oxide is possessed many applications in specific diseases as well as optimized $\mathrm{Bi}_{2} \mathrm{O}_{3}$ nanostructures for electrical, medical, biological sensors, and other relevant applications. It provides science (details) about the trapped charged recombination sites related to metastable defects in the lattice based on whether the entrapping process is due to heat. The catalytic behavior of $\mathrm{Bi}_{2} \mathrm{O}_{3}$ is also influenced by the presence of an oxygen vacancy. Thus, small variation in the lattice structure due to the presence of inclusion impurities, substituted ions, surface defects in ppm concentration reveals successful degradation of photocatalytic properties. A number of studies $\mathrm{Bi}_{2} \mathrm{O}_{3}$ have been carried out on reaction optimization and structural modification (e.g., 
metal doping or material hybridization) in order to improve the photoactivity and energy conservation.

The complex degradation behaviour of natural organic matter (NOM) was investigated using photocatalytic oxidation systems with a novel catalyst based on a hybrid composite of zinc-bismuth oxides and $\mathrm{g}-\mathrm{C}_{3} \mathrm{~N}_{4}(\mathrm{ZBO}-\mathrm{CN})$. by Hai Bang Truong et al. Under low-intensity visible light irradiation, the photooxidation procedure effectively removed NOM, with removal rates of 53-74\% and $65-88 \%$ respectively, based on dissolved organic carbon (DOC) and UV absorption coefficient (UV254) at $1.5 \mathrm{~g} / \mathrm{L}$ of catalyst [2].

Variety of synthesis techniques have already been developed to produce $\mathrm{Bi}_{2} \mathrm{O}_{3}$ in powder and thin film form, its various properties strongly depend on its structure including the crystal size, orientation, morphology and density. The controlled synthesis of monodisperse $\mathrm{Bi}_{2} \mathrm{O}_{3}$ nanoparticles remains a challenge. Chemical synthesis is a straight forward synthesis route and the temperature is the key parameter which effectively hydrolyses to starting solution for the well-defined nano scaled $\mathrm{Bi}_{2} \mathrm{O}_{3}$. The photocatalytic degradation of dyes in aqueous solution is shown by the synthesis of $\mathrm{Bi}_{2} \mathrm{O}_{3}$ NPs using a high yield approach Among all the synthesis routes, hydrothermal synthesis should be further improved, to meets the requirement of environmental production [6].

Methylene blue (MB) is a water-soluble dye which widely used in pharmaceuticals, food industries and textile printing. The safe removal of MB dye is the prime aim of our present study. This study concentrates to examine the effect of $\mathrm{pH}$ value and the reaction of photocatalytic performance by using $\mathrm{Bi}_{2} \mathrm{O}_{3}$ NPs was reported here. They removal of dye to decompose by the photocatalytic process explains the separation of photogenerated charge carriers for enhancing the photocatalytic activity. However, micron grain sized $\mathrm{Bi}_{2} \mathrm{O}_{3}$ which act as a semiconductor so its surface area is very low and photogenerated charge carriers cannot be transferred for the fast charge carrier's recombination. 
In this work, we report the synthesis of photocatalytic activity of $\mathrm{Bi}_{2} \mathrm{O}_{3}-\mathrm{NPs}$ structure studied by hydrothermal process. X-ray diffraction (XRD), energy dispersive x-ray spectroscopy (EDXS), Field Emission scanning electronmicroscope (FE-SEM), transmission electron microscope (TEM), UV-VIS-NIR spectrophotometer and Fourier transfer infrared spectroscopy (FTIR) was used to confirm and exhibits the different physical properties of $\mathrm{Bi}_{2} \mathrm{O}_{3}-\mathrm{NPs}$. The photocatalytic response of as-synthesized $\mathrm{Bi}_{2} \mathrm{O}_{3}-\mathrm{NPs}$ as promising photocatalyst was checked using the degradation of Methylene blue (MB) under irradiation of LED white light also examined the effect of crystallite size of the catalyst on (MB) dye concentration prepared by hydrothermal synthesis using organic pollutants such as phenol, 4chloro phenol, and 4-nitro phenol in water [1].

\section{Experimental methods}

\subsection{Synthesis of $\mathrm{Bi}_{2} \mathrm{O}_{3} \mathrm{Nano}$ particles}

Nano particles were synthesized using analytical grade Bismuth (III) nitrate (Bi $\left(\mathrm{NO}_{3}\right)_{3} \cdot 6 \mathrm{H}_{2} \mathrm{O}$, Sigma Aldrich,99\%), sodium hydroxide (NaOH, Sigma Aldrich,99\%) and Nitric acid (Sigma Aldrich,68\%) without further purification and Ultra-pure water. In a hydrothermal synthesis, $1 \mathrm{mmol}$ of $\mathrm{Bi}\left(\mathrm{NO}_{3}\right)_{3} \cdot 6 \mathrm{H}_{2} \mathrm{O}$ was dissolved in $50 \mathrm{~mL}$ of $0.3 \mathrm{M}$ Nitric acid, then the solution was sonicated about 15 minutes, at room temperature with purpose to achieve homogeneous solution. Then, $0.2 \mathrm{M}$ of sodium hydroxide solution of 5-6 mmol (1:5 to 1:6 molar ratio of $\mathrm{Bi}\left(\mathrm{NO}_{3}\right)_{3} .5 \mathrm{H}_{2} \mathrm{O}$ to $\left.\mathrm{NaOH}\right)$ was added drop by drop into the clear solution under vigorous stirring.

During the reaction, the $\mathrm{pH}$ of the mixture increases gradually and attained approximately above 10 , precipitation process started to form white precipitate. After 30 min continuous stirring, white precipitate obtained was transferred into autoclave with Teflon lining and kept at $160^{\circ} \mathrm{C}$ for 12 hours for hydrothermal treatment. The autoclave was cooled to room temperature naturally. The yellow precipitate was obtained by centrifugation ( 6 min 
with $7500 \mathrm{rpm}$ ) and washed several times with ultra-pure water and dried at $80^{\circ} \mathrm{C}$ for 12 hours. Finally, the products were calcined at $350^{\circ} \mathrm{C}$ for 3 hours for further characterization $[8]$.

\subsection{Characterization}

The crystalline properties of the synthesized $\mathrm{Bi}_{2} \mathrm{O}_{3}-\mathrm{NPs}$ were studied by XRD using a Bruker (D5005) X-ray diffractometer equipped with graphite monochromatized $\mathrm{CuK} \alpha$ radiation $(\lambda=1.54056 \AA)$. An accelerating voltage of $40 \mathrm{kV}$ and emission current of $30 \mathrm{~mA}$ were adopted for the measurements. In addition to XRD, FTIR spectroscopy measurements were also performed to confirm the structure of the $\mathrm{Bi}_{2} \mathrm{O}_{3}$-NPs. The chemical composition of $\mathrm{Bi}_{2} \mathrm{O}_{3}$-NPs were studied using SEM. The SEM measurements were performed by a Hitachi S-4800 high resolution (HR) field emission scanning electron microscope. The FE-SEM equipment was also furnished with an EDAX spectrometer that was used for elemental analysis. Absorption spectra of the samples in the diffused reflectance spectrum (DRS) mode were recorded in the wavelength range of 200- $1000 \mathrm{~nm}$ using a spectrophotometer (Jasco V 670), with $\mathrm{BaSO}_{4}$ as a reference. From the adsorption edge, the band gap values were calculated by extrapolation.

\subsection{Photocatalytic Activity}

Photocatalytic activity of synthesized $\mathrm{Bi}_{2} \mathrm{O}_{3}$-NPs were examined by the rate of degradation of MB under the effect LED white light irradiation. All photo catalytic reaction were performed out in photo catalytic reactor system, which consists of a cylindrical borosilicate glass reactor vessel with volume of $250 \mathrm{~mL}$, a cooling water jacket, and a LED white light, Institute of Electric Light Source, Beijing) positioned axially at the center as a visible light. The reaction temperature was kept at $25^{\circ} \mathrm{C}$ by circulating the cooling water. A special glass frit as an air diffuser was fixed at the reactor to uniformly disperse air into the solution. For each run the reaction suspension was freshly prepared by adding $0.250 \mathrm{~g}$ of 
catalyst into $250 \mathrm{~mL}$ of initial concentration of $5 \mathrm{mg} / \mathrm{L}$ of MB. After the degradation reaction, filtration was done for all samples using syringe and syringe filter $0.45 \mu \mathrm{m}$ to remove any precipitated particles. The filtrate was analysed by an UV-Vis spectrometer (UC-2450SHIMADZU). The maximum characteristic absorption wavelength of $\mathrm{MB}$ was positioned [9].

\section{Result and Discussion}

\subsection{XRD analysis}

The phase crystallinity and purity of hydrothermally synthesized $\mathrm{Bi}_{2} \mathrm{O}_{3}-\mathrm{NPs}$ sample were investigated using XRD analysis. Figure 1 exhibits the X-ray diffraction pattern of $\mathrm{Bi}_{2} \mathrm{O}_{3}$ materials exhibited reflection peaks at 31.923 of glancing angle. All reflection peaks can be well indexed with a pure tetragonal phase of crystalline $\mathrm{Bi}_{2} \mathrm{O}_{3}$, which are in good agreement with the fiber structure of tetragonal phase (JCPDS card No: 65-4028). The broad reflection peaks suggested that the materials are nano crystalline structure. The crystalline of $\mathrm{Bi}_{2} \mathrm{O}_{3} \mathrm{NPs}$ is nearly $42 \mathrm{~nm}$, which shows that the product consists of needle shaped nano crystals. The additional reflection peaks indicates that when $\mathrm{OH}$ ions are used for the preparation of nano crystalline structure with additional phase of $\mathrm{Bi}_{2} \mathrm{O}_{3}$ is strongly influenced by the variation of $\mathrm{pH}$ solution caused by particular molar ratio of $\mathrm{NO}_{3}{ }^{-}$and hydroxyl ions. In our case the high concentration of reducing agent $\mathrm{NaOH}$ which is used to raise the $\mathrm{pH}$ value of given solution between 8 to 10 to reach the optimum condition [10]. The temperature of the hydrothermal synthesis increasing concentration of aqueous solution and $\mathrm{Bi}^{3+}$ ions species into pure obtained phase suggesting that this $160^{\circ} \mathrm{C}$ optimum temperature of the solvent are used for stabilizing the agent. The materials exhibited crystalline structure of tetragonal with the lattice constants $3.850 \mathrm{~A}^{\mathrm{o}}$ and $12.250 \mathrm{~A}^{\mathrm{o}}$ which is good agreement with JCPDS No 65-4028. Calculated structural parameters were tabulated in Table 1 [11] 
Average crystallite size was determined from predominant XRD peak using Scherrer Eq. (1)

$$
D=\frac{k \lambda}{\beta \cos \theta}
$$

where $\mathrm{k}=0.9$ the numerical shape factor which is a constant,$D$ is crystallite size, $\lambda$ is wavelength of incident radiation, $\beta$ is the FWHM in radians, and $\theta$ is Bragg angle taken in radians Dislocation density $(\delta)$ was calculated from crystallite size using Eq.(2)

$$
\delta=\frac{1}{D^{2}}
$$

The lattice constants a and c are calculated for tetragonal Structures

$$
a=2.8284 * d_{\text {spacing }} \quad \frac{1}{d^{2}}=\frac{h^{2}+k^{2}}{a^{2}}+\frac{l^{2}}{c^{2}}
$$

Where $\mathrm{d}$ is interplanar spacing, $\mathrm{h}, \mathrm{k}, \mathrm{l}=$ miller indices

\subsection{XPS analysis}

The XPS spectra of $\mathrm{Bi}_{2} \mathrm{O}_{3}$ NPs were obtained and shown in figure 2(a). There are two asymmetrical peaks observed at $292 \mathrm{eV}$ and $198 \mathrm{eV}$ correspond to $\mathrm{Bi} 4 \mathrm{f}$ orbitals band its electro chemical reduction of new peaks was consistent with $\mathrm{Bi} 4 \mathrm{f}$ spectra for the Bi metal. This peak at $534 \mathrm{eV}$ correspond to $\mathrm{Bi}$ ions and its signal voltage is assigned to $\mathrm{Bi}^{3+}$ ion which are in good agreement with reported XPS analysis of $\mathrm{Bi}_{2} \mathrm{O}_{3}$ NPs. The figure 2(b) exhibited the two peaks at $173 \mathrm{eV}$ and $168 \mathrm{eV}$ can be assigned to $\mathrm{Bi} 4 \mathrm{f} 5 / 2$ and $\mathrm{Bi} 4 \mathrm{f} 3 / 2$, respectively. The surface analysis of $\mathrm{Bi}_{2} \mathrm{O}_{3}$ NPs and its peaks were resulted there is no shoulder of $\mathrm{Bi} 4 \mathrm{f}_{7 / 2}$ peaks related to either bivalent or tetravalent states, instead it gives $\mathrm{Bi} 4 \mathrm{f}_{5 / 2}$ of $\mathrm{Bi}_{2} \mathrm{O}_{3}$ gives a report as the surface of the prepared particle were consist of pure $\mathrm{Bi}_{2} \mathrm{O}_{3}$ and they were not any $\mathrm{BiO}$ and $\mathrm{Bi}_{2} \mathrm{O}_{5}$ phases [12]. The spectrum for the $\mathrm{O}$ 1s field is shown in Figure 2(c). A 
low-intensity signal is seen, indicating there is not any oxygen in this sample. It's possible that the presence of oxygen in the samples is due to adsorption from the surrounding moisture.The valence band offset $\Delta v$ can be described by the formula,

$$
\Delta E_{v}=\left(4 f-V_{B M}\right)+\Delta E_{C L}
$$

$\mathrm{CL}=$ core level

$\Delta \mathrm{ECL}$

$\Delta E_{C L}=$ Energy difference between $\mathrm{Bi} 4 \mathrm{f}\left(\mathrm{Bi}_{2} \mathrm{O}_{3}\right)$ core levels which are measured on this sample by XPS spectra. $V_{\mathrm{BM}}$ position in the Valence band spectra was determined from the analysis and its values are $2.74 \mathrm{eV}$ to $2.42 \mathrm{eV}$ respectively which is fine comparable with the results of bandgap of $\mathrm{Bi}_{2} \mathrm{O}_{3}$ which is shown in the figure 5(a) and (b). The linear extrapolation of the leading edges of valence band spectra were determined according to linear extrapolation so the leading edges of the valence band spectra recorded to the base lines [13].

\subsection{EDAX and SEM analysis}

The elemental and compositional properties of the sample, of $\mathrm{Bi}_{2} \mathrm{O}_{3}$ NPs were studied by EDAX. The EDAX spectra of hydrothermal synthesized $\mathrm{Bi}_{2} \mathrm{O}_{3}$-NPs with (1:5) and (1:6) molar ratios are shown in figure $3(\mathrm{a})$ and (b) indicates. The table 2 represent the quantitative EDAX analysis, it indicates that the atomic ratios of $\mathrm{Bi}: \mathrm{O}$ element for the proportion ratio (1:6) and (1:5) respectively. From the figure only isolated sub- micron spherical structure with average diameter of $2 \mu \mathrm{m}$ are observed. The appearance of spherical distribution could be attributed to the formation of coordination compounds of bismuth and oxygen ions

The figure 4 exhibited the morphological study of the prepared $\mathrm{Bi}_{2} \mathrm{O}_{3} \mathrm{NPs}$. The rate of change of nucleation and crystal formation was rationally concluded that the surfactant and oxidizing agent an important key factor for the formation of fiber like structure [14]. A perfect geometrical shape and well-defined boundary of the structure are the important parameters that could be measured with the different variation of concentration of $\mathrm{Bi}_{2} \mathrm{O}_{3}$. So 
that the morphology obtained are treated with different variation of concentration of source materials.

\subsection{UV DRS analysis}

The UV-Visble studies of two different concentrations of the samples were carried out for the calculation of bandgap values using Tauc plots. The absorption edge for the two samples is different and was nearly 2.74 and $2.42 \mathrm{eV}$, it influenced the variation of crystallite size. The direct bandgap was carried out for the allowed transition to study the absorption coefficient, percentage of transition and the bandgap value. The figure 5(a)\& (b) represents the typical plot of the wavelength vs absorption coefficient $(\alpha h v)^{2}$ and wavelength vs percentage of transmittance. It represents the allowed transition of $\mathrm{Bi}_{2} \mathrm{O}_{3}$ for the two different molar ration with proposed semiconductor behavior of prepared nano particles [15].

At the same time the Fermi level of two samples is related to the oxygen pressure due to using DRS reflectance spectra. For an integrating sphere exclude the diffuse scattering effects. The intending band alignment would always be a dominant behavior to study the band alignment between bismuth and oxygen ions. It is worthwhile to known that the surface area is nearly $25 \mathrm{~m}^{2} / \mathrm{g}$ for the two samples which results in decreasing crystallite size of phase transformation, this phase for these two samples shows a thermodynamically spontaneous transformation for the design and optimum surface area of the samples are 2.74 and $2.42 \mathrm{eV}$ [16] .

\subsection{FTIR analysis}

The obtained $\mathrm{Bi}_{2} \mathrm{O}_{3}$ NPs was studied by infrared spectroscopy FTIR. Figure 6 shows the obtained infrared spectra. The phase composition and the vibrational frequency were formed by using FTIR spectroscopy $\mathrm{Bi}\left(\mathrm{NO}_{3}\right)_{3}$ which can be transformed into single phase $\mathrm{Bi}_{2} \mathrm{O}_{3}$ and its $\mathrm{OH}$ group was released by the oxidation reactions of reagent dissolved in the preparation. The distilled water $\mathrm{OH}$ group was observed for the influence of composition 
of reaction products containing large amounts of hydroxyl group with high specific surface area $25 \mathrm{~m}^{2} \mathrm{~g}^{-1}$ were treated at the $\mathrm{pH}$ condition of 6 . Suppose the $\mathrm{pH}$ is greater than 7 the IR region showed no $\mathrm{OH}$ group in the products the morphology of the product of fiber like structure inferred, the particles were prepared under acidic condition [17].

The crystalline product containing bismuth and oxygen ions having fibre shaped particles resulted a possible reaction mechanism by hydration and were followed by hydrolysis. The formation of $\mathrm{Bi}_{2} \mathrm{O}_{3}$ and the condensation of the $\mathrm{OH}$ group is strongly influenced by the $\mathrm{pH}$ of the reaction medium. The accelerated $\mathrm{Bi}^{3+}$ ion causes non conventional volume of reaction by the applied temperature inside the chamber [18].

\subsection{Photocatalytic performance}

The figure 8(a) and (b) depicts temporal changes in the UV-Vis spectra of MB in photodegradation reaction with prepared $\mathrm{Bi}_{2} \mathrm{O}_{3}$ NPs of different molar ratios. It is found that only about $10 \% \mathrm{MB}$ was degraded after 30 minutes degradation with photocatalyst in the absence of visible light irradiation. The figure 8(a) represents the $\mathrm{Bi}_{2} \mathrm{O}_{3}$ NPs e of 1:5 molar ratio with a bandgap of $\mathrm{Bi}_{2} \mathrm{O}_{3}$ (1-5 bandgap $2.74 \mathrm{eV}$ and 1-6 bandgap $2.42 \mathrm{eV}$ ) cannot be excited in the absence of visible light irradiation, the photodegradation of MB over 1:5 molar ratio of $\mathrm{Bi}_{2} \mathrm{O}_{3}$ in the absence of visible light irradiation shows negligible efficiency. Noticeably, 1:6 ratio displays low efficiency for MB degradation due to its low valence band location, although it has been considered an excellent visible-light-driven photocatalyst [19]. The suggested photocatalytic reaction mechanism for the degradation of MB dye over $\mathrm{Bi} 2 \mathrm{O} 3$ NPs is depicted in figure 9. The volume of photocatalyst suspended in $100 \mathrm{~mL}$ of $\mathrm{MB}$ solution was determined using the atypical behavior test [20]. After allowing the suspension to achieve adsorption equilibrium in the dark for 2 hours, the photocatalytic reaction was started under visible light up to $250^{\circ} \mathrm{C}$. The studies were conducted out in a pyrex cylindrical photoreactor $(\mathrm{ID}=2.8 \mathrm{~cm})$. 
with an air distributor system (Qair=150 $\mathrm{cm} 3 / \mathrm{min}(\mathrm{STP})$ ), a magnetic stirrer to keep the photocatalyst suspended in the aqueous solution, and a temperature controller

A strip of 30 white light LEDs (nominal power: $6 \mathrm{~W}$ ) with wavelength emission in the range 400-800 nm or an equivalent number of blue light LEDs (nominal power: $6 \mathrm{~W}$ ) with wavelength emission in the range $400-550 \mathrm{~nm}$ was irradiated in the photoreactor. The LED strip was wrapped around the reactor to ensure that the light source illuminated the reaction volume evenly. Figure 8 shows a graphical diagram of Bi2O3 NPs, which shows the degradation principle, as well as the fraction of the curve colored in blue, which shows the spectral emission of blue LEDs. A schematic of the photocatalytic reactor is seen on the left side of the same figure. Slurry samples were gathered at predetermined times and centrifuged at $4000 \mathrm{rpm}$ for 20 minutes for removing photocatalyst particles. The centrifuged samples were analysed to determine the change of dyes concentration, measured with a Perkin Elmer $\mathrm{UV}-\mathrm{V}$ is spectrophotometer at $663 \mathrm{~nm}[21]$.

As photodegradation of organic pollutant over $\mathrm{Bi}_{2} \mathrm{O}_{3}$ nanoparticles was dominated as the hole oxidation process, the photogenerated holes over $\mathrm{Bi}_{2} \mathrm{O}_{3}$ did not exhibit enough over potential for the oxidation of $\mathrm{MB}$, thus leading to the low photocatalytic efficiency. Importantly, it is found that the entire two ratios of the samples exhibit remarkably enhanced photocatalytic activities for $\mathrm{MB}$ degradation as compared to these as prepared samples. Among them, $\mathrm{Bi}_{2} \mathrm{O}_{3}$ nanoparticles with 1:5 molar ratio shows the highest photocatalytic activity and could approximately $80 \%$ degrade $\mathrm{MB}$ within $3 \mathrm{~h}$. It is believed that $\mathrm{Bi}_{2} \mathrm{O}_{3}(1: 5)$ ratio lattice varies the location of the valence band somehow; therefore, the photogenerated holes possess higher oxidation power for MB degradation [22].

In the dark Conditions the concentration of MB decreased deuring the firs hour of the test and it was remaining constant in the second hour, indicating that the dye adsorption of equilibrium of on catalyst surface had been reached. The curves show that $\mathrm{Bi}_{2} \mathrm{O}_{3}$ catalysts 
have different amounts of MB adsorbed in dark [23]. To explain this last result, the specific area was estimated for the sample (1-5) it was $25 \mathrm{~m}^{2} / \mathrm{g}$, while for (1-6) it was $23 \mathrm{~m}^{2} / \mathrm{g}$. As seen in figure 10, the volume of organic dye adsorbed increases as the actual surface area increases, roughly proportional to the differences in the area values.

Since the solution was exposed to visible light after the dark time, the reaction began to take place. Figure 10 shows that $\mathrm{Bi}_{2} \mathrm{O}_{3}$ NP is slightly effective for $\mathrm{MB}$ decolourization, the / reduction being about $10 \%$, a value similar to that of photolysis reaction (14\%). On the contrary, different proportions of $\mathrm{Bi}_{2} \mathrm{O}_{3}$ NP photocatalysts exhibited higher photocatalytic activity under visible light irradiation. After 250 minutes, the decolorization activity of Bi2O3 NPs was in the following order: $(1: 5)>(1: 6)$. The dye concentration decides the real value of MB conversion after the dark time. The decolorization of MB does not always lead to the molecule's oxidation and mineralization; in particular, the reduced form of MB can be formed in the presence of light [24].

\section{Conclusion}

In the present work the $\mathrm{Bi}_{2} \mathrm{O}_{3}$ NPs was successfully synthesized by the efficient hydrothermal method with two different molar ratio of precipitating agent of $\mathrm{NaOH}$ which two samples (1:5) and (1:6). The prepared $\mathrm{Bi}_{2} \mathrm{O}_{3}$ NPs characterized and tested in the photodegradation of MB. The crystal size was observed well spherical and also fiber shaped as 30.7 and $42.84 \mathrm{~nm}$. The optical properties were characterized by using UV-Vis spectrometer and its band gap value was calculated as $2.74 \mathrm{eV}$ and $2.42 \mathrm{eV}$ respectively. Morphological studies were obtained by using scanning electron microscopy and the elemental X-ray analysis confirmed the proportions of $\mathrm{Bi}_{2} \mathrm{O}_{3}$ nanoparticles of two samples (1:5) and (1:6). FTIR Spectrometer studies explained the condensation of $\mathrm{OH}$ group accompanied by the formation of $\mathrm{Bi}_{2} \mathrm{O}_{3}$ which is strongly dependent on the $\mathrm{pH}$ of reaction. $\mathrm{A}$ lattice change was observed, symmetric and asymmetric bonds were analysed by using FTIR 
spectrometer. The binding energy and its energy level were analysed by using X-ray Photo electron Spectroscopy and its value was 292 and $534 \mathrm{eV}$. These hydrothermally prepared $\mathrm{Bi}_{2} \mathrm{O}_{3}$ NPs are efficient in the degradation of a standard pollutant, MB , under the irradiation of LED white light.

\section{Availability of data and material:}

The data that support the findings of this study are available in https://doi.org/10.1016/j.jtusci.2015.01.009"and https://doi.org/10.1016/j.jtusci.2015.01.009

\section{References}

[1] Patil, M.M., Deshpande, V.V., Dhage, S.R. and Ravi, V., 2005. Synthesis of bismuth oxide nanoparticles at 100 C. Materials letters, 59(19-20), pp.2523-2525.

[2] Truong, H.B., Huy, B.T., Ly, Q.V., Lee, Y.I. and Hur, J., 2019. Visible light-activated degradation of natural organic matter (NOM) using zinc-bismuth oxides-graphitic carbon nitride (ZBO-CN) photocatalyst: Mechanistic insights from EEMPARAFAC. Chemosphere, 224, pp.597-606.

[3] Police, A.K.R., Vattikuti, S.P., Mandari, K.K., Chennaiahgari, M., Sharma, P., Valluri, D.K. and Byon, C., 2018. Bismuth oxide cocatalyst and copper oxide sensitizer in $\mathrm{Cu}_{2} \mathrm{O} / \mathrm{TiO}_{2} / \mathrm{Bi}_{2} \mathrm{O}_{3}$ ternary photocatalyst for efficient hydrogen production under solar light irradiation. Ceramics International, 44(10), pp.11783-11791

[4] Vinoth, R., Babu, S.G., Ramachandran, R. and Neppolian, B., 2017. Bismuth oxyiodide incorporated reduced graphene oxide nanocomposite material as an efficient photocatalyst for visible light assisted degradation of organic pollutants. Applied Surface Science, 418, pp.163-170.

[5] Hernandez-Delgadillo, R., Velasco-Arias, D., Martinez-Sanmiguel, J.J., Diaz, D., Zumeta-Dube, I., Arevalo-Niño, K. and Cabral-Romero, C., 2013. Bismuth oxide aqueous colloidal nanoparticles inhibit Candida albicans growth and biofilm formation. International journal of nanomedicine, 8, p.1645.

[6] Chai, S.Y., Kim, Y.J., Jung, M.H., Chakraborty, A.K., Jung, D. and Lee, W.I., 2009. Heterojunctioned $\mathrm{BiOCl} / \mathrm{Bi}_{2} \mathrm{O}_{3}$, a new visible light photocatalyst. Journal of Catalysis, 262(1), pp.144-149. 
[7] Ling, C.D., 1999. Structural relationships among bismuth-rich phases in the $\mathrm{Bi}_{2} \mathrm{O}_{3}-$ $\mathrm{Nb}_{2} \mathrm{O}_{5}, \mathrm{Bi}_{2} \mathrm{O}_{3}-\mathrm{Ta}_{2} \mathrm{O}_{5}, \mathrm{Bi}_{2} \mathrm{O}_{3}-\mathrm{MoO}_{3}$, and $\mathrm{Bi}_{2} \mathrm{O}_{3}-\mathrm{WO}_{3}$ systems, Journal of Solid State Chemistry, 148(2), pp.380-405

[8] Jha, R.K., Pasricha, R. and Ravi, V., 2005. Synthesis of bismuth oxide nanoparticles using bismuth nitrate and urea. Ceramics international, 31(3), pp.495-497.

[9] Mallahi, M., Shokuhfar, A., Vaezi, M.R., Esmaeilirad, A. and Mazinani, V., 2014. Synthesis and characterization of bismuth oxide nanoparticles via sol-gel method. AJER, 3(4), pp.162-5.

[10] Krishna Reddy, J., Srinivas, B., Durga Kumari, V. and Subrahmanyam, M., 2009. $\mathrm{Sm}^{3+}$-doped $\mathrm{Bi}_{2} \mathrm{O}_{3}$ photocatalyst prepared by hydrothermal synthesis. Chem Cat Chem, 1(4), pp.492-496.

[11] M. Rivenet, P. Roussel, F. Abraham One-dimensional inorganic arrangement in the bismuth oxalate hydroxide Bi(C2O4)OH J. Solid State Chem., 181 (2008), p. 2586

[12] Chai, S.Y., Kim, Y.J., Jung, M.H., Chakraborty, A.K., Jung, D. and Lee, W.I., 2009. Heterojunctioned $\mathrm{BiOCl} / \mathrm{Bi}_{2} \mathrm{O}_{3}$, a new visible light photocatalyst. Journal of Catalysis, 262(1), pp.144-149

[13] Drache, M., Roussel, P. and Wignacourt, J.P., 2007. Structures and oxide mobility in $\mathrm{Bi}-\mathrm{Ln}-\mathrm{O}$ materials: heritage of $\mathrm{Bi}_{2} \mathrm{O}_{3}$. Chemical reviews, 107(1), pp.80-96.

[14] Saritha, D., Markandeya, Y., Salagram, M., Vithal, M., Singh, A.K. and Bhikshamaiah, G., 2008. Effect of $\mathrm{Bi}_{2} \mathrm{O}_{3}$ on physical, optical and structural studies of $\mathrm{ZnO}-\mathrm{Bi}_{2} \mathrm{O}_{3}-\mathrm{B}_{2} \mathrm{O}_{3}$ glasses. Journal of Non-Crystalline Solids, 354(52-54), pp.55735579

[15] Saritha, D., Markandeya, Y., Salagram, M., Vithal, M., Singh, A.K. and Bhikshamaiah, G., 2008. Effect of $\mathrm{Bi}_{2} \mathrm{O}_{3}$ on physical, optical and structural studies of $\mathrm{ZnO}-\mathrm{Bi}_{2} \mathrm{O}_{3}-\mathrm{B}_{2} \mathrm{O}_{3}$ glasses, Journal of Non-Crystalline Solids, 354(52-54), pp.55735579

[16] Pan, C., Li, X., Wang, F. and Wang, L., 2008. Synthesis of bismuth oxide nanoparticles by the polyacrylamide gel route. Ceramics international,34(2), pp.439-441.

[17] Barreca, D., Morazzoni, F., Rizzi, G.A., Scotti, R. and Tondello, E., 2001. Molecular oxygen interaction with $\mathrm{Bi}_{2} \mathrm{O}_{3}$ : a spectroscopic and spectromagnetic investigation. Physical Chemistry Chemical Physics, 3(9), pp.1743-1749

[18] Hwang, G.H., Han, W.K., Kim, S.J., Hong, S.J., Park, J.S., Park, H.J. and Kang, S.G., 2009. An electrochemical preparation of bismuth nanoparticles by reduction of 
bismuth oxide nanoparticles and their application as an environmental sensor. Journal of Ceramic Processing Research, 10(2), pp.190-194..

[19] Bessekhouad, Y., Robert, D. and Weber, J.V., 2005. Photocatalytic activity of $\mathrm{Cu}_{2} \mathrm{O} / \mathrm{TiO}_{2}, \mathrm{Bi}_{2} \mathrm{O}_{3} / \mathrm{TiO}_{2}$ and $\mathrm{ZnMn}_{2} \mathrm{O}_{4} / \mathrm{TiO}_{2}$ heterojunctions. Catalysis Today, 101(34), pp.315-321

[20] Li, R., Chen, W., Kobayashi, H. and Ma, C., 2010. Platinum-nanoparticle-loaded bismuth oxide: an efficient plasmonic photocatalyst active under visible light. Green Chemistry, 12(2), pp.212-215.

[21] El-Batal, A.I., El-Sayyad, G.S., El-Ghamry, A., Agaypi, K.M., Elsayed, M.A. and Gobara, M., 2017. Melanin-gamma rays assistants for bismuth oxide nanoparticles synthesis at room temperature for enhancing antimicrobial, and photocatalytic activity. Journal of Photochemistry and Photobiology B: Biology, 173, pp.120-139.

[22] Li, X., Sun, Y., Xiong, T., Jiang, G., Zhang, Y., Wu, Z. and Dong, F., 2017. Activation of amorphous bismuth oxide via plasmonic Bi metal for efficient visiblelight photocatalysis. Journal of catalysis, 352, pp.102-112.

[23] Xiong, M., Chen, L., Yuan, Q., He, J., Luo, S.L., Au, C.T. and Yin, S.F., 2015. Controlled synthesis of graphitic carbon nitride/beta bismuth oxide composite and its high visible-light photocatalytic activity. Carbon, 86, pp.217-224.

[24] Lin, G., Tan, D., Luo, F., Chen, D., Zhao, Q., Qiu, J. and Xu, Z., 2010. Fabrication and photocatalytic property of $\alpha$-Bi2O3 nanoparticles by femtosecond laser ablation in liquid. Journal of Alloys and Compounds, 507(2), pp. L43-L46. 
Figures

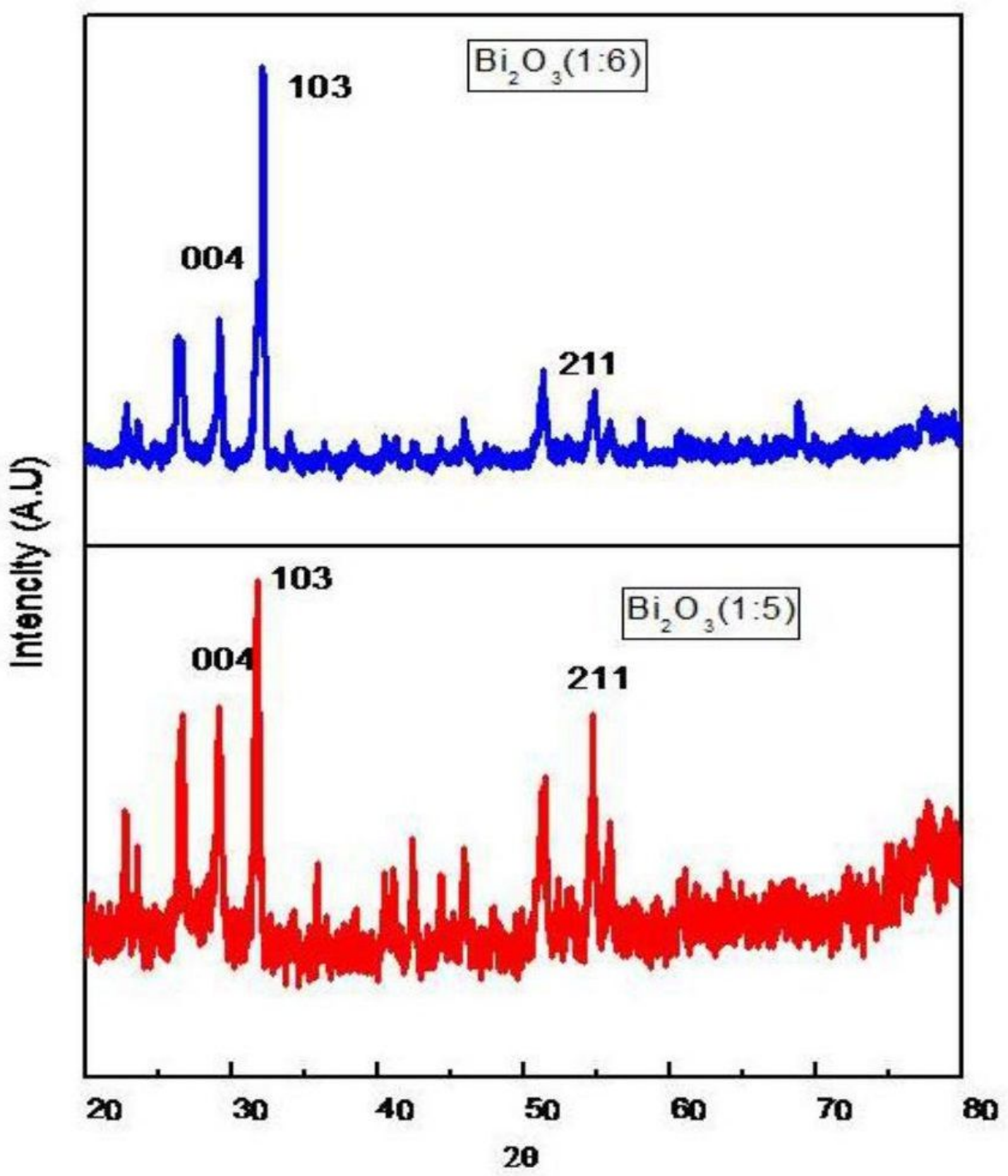

Figure 1

XRD Analysis of Bi203 NPs with different molar ratio 

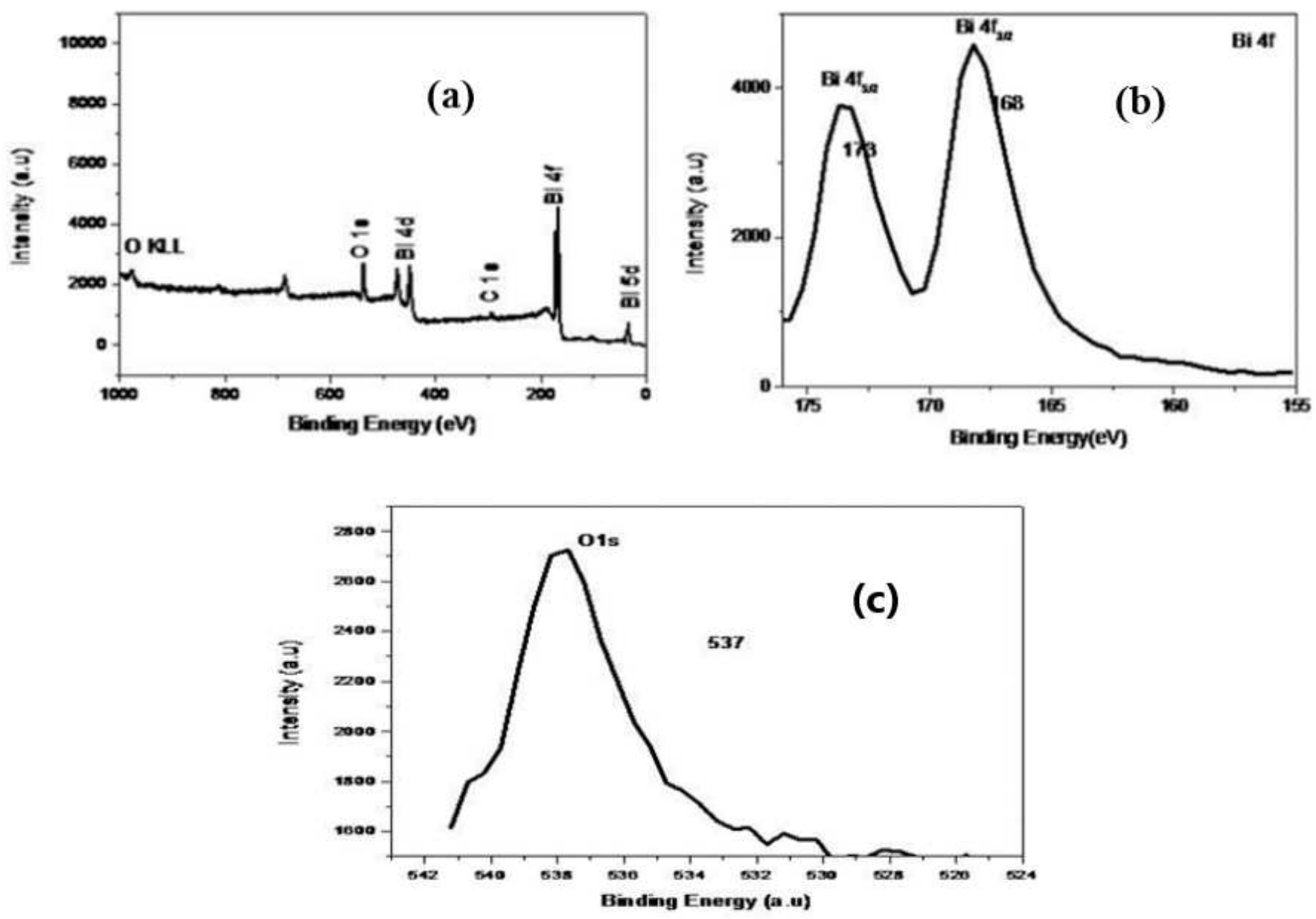

Figure 2

(a) XPS Analysis of Bi2O3 (1:6) (b) XPSspectra of the Bi-4f (c) XPS spectra of the 01 s region 

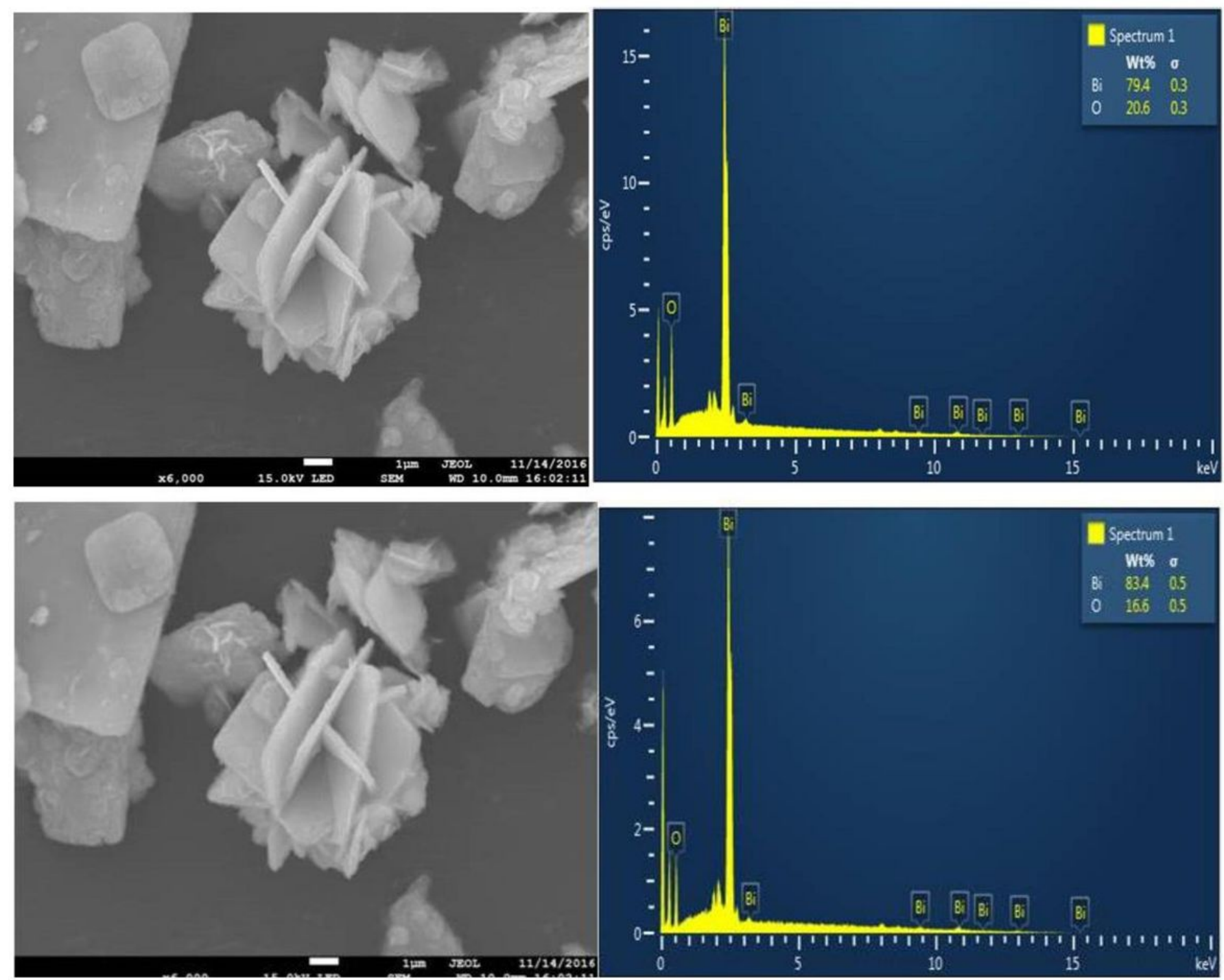

Figure 3

SEM analysis of Bi2O3 Nanoparticles for the proportion ratio (1:6) and (1:5) 

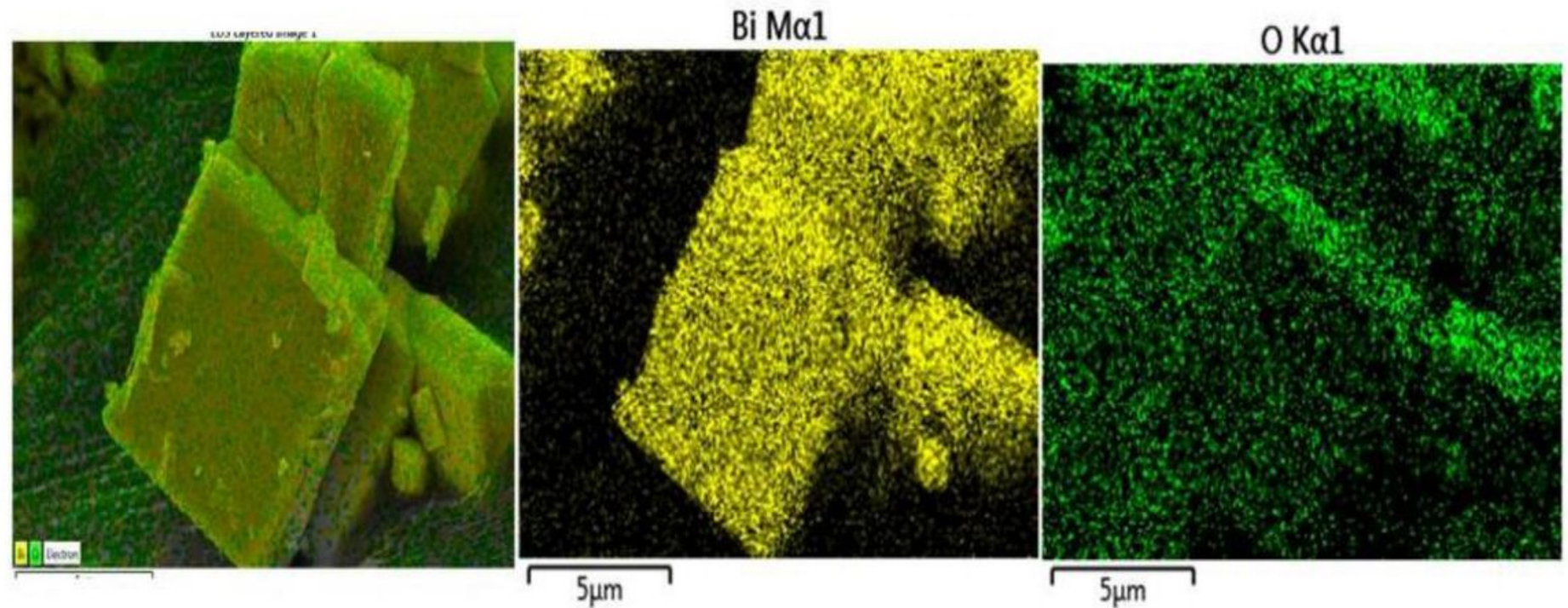

Figure 4

SEM mapping of Bi203 NPs proportion ratio of (1:5) and (1:6)
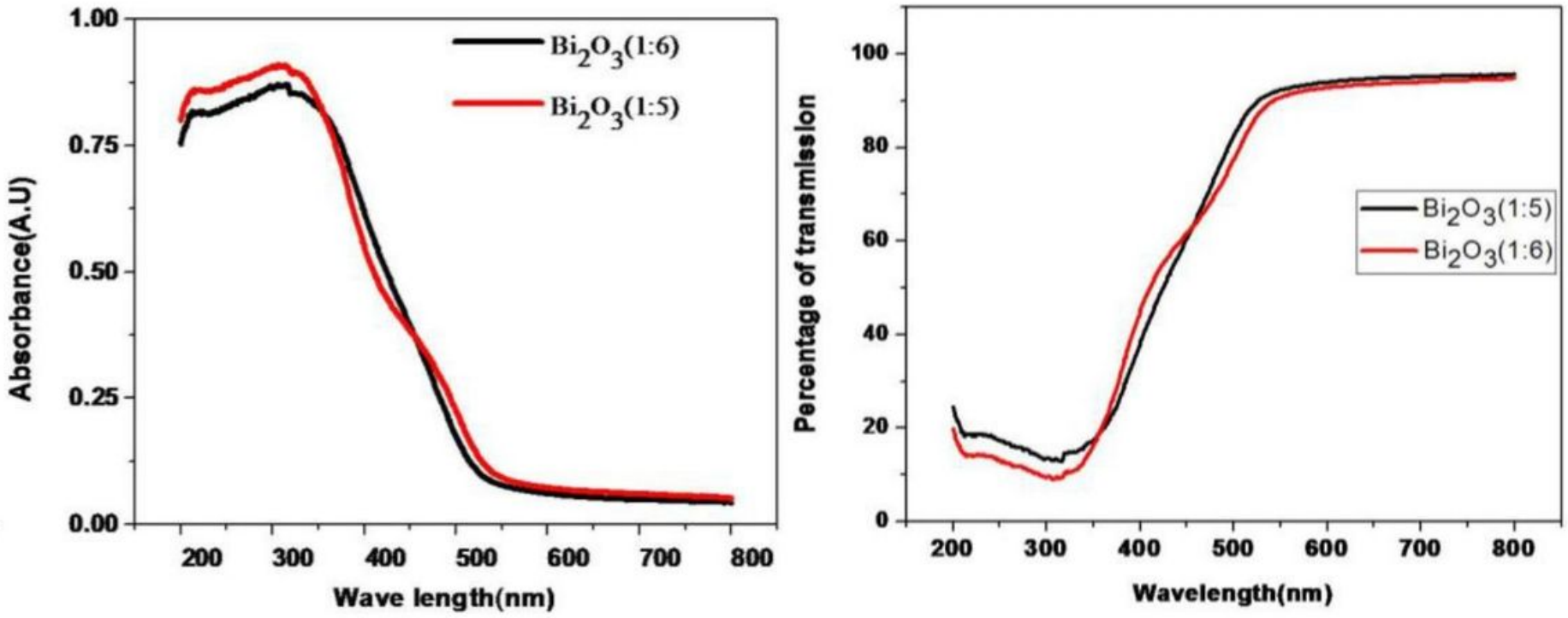

Figure 5

(a) Absorbance curve (b) Transmittance curve of Bi203 (1:5) and (1:6) 

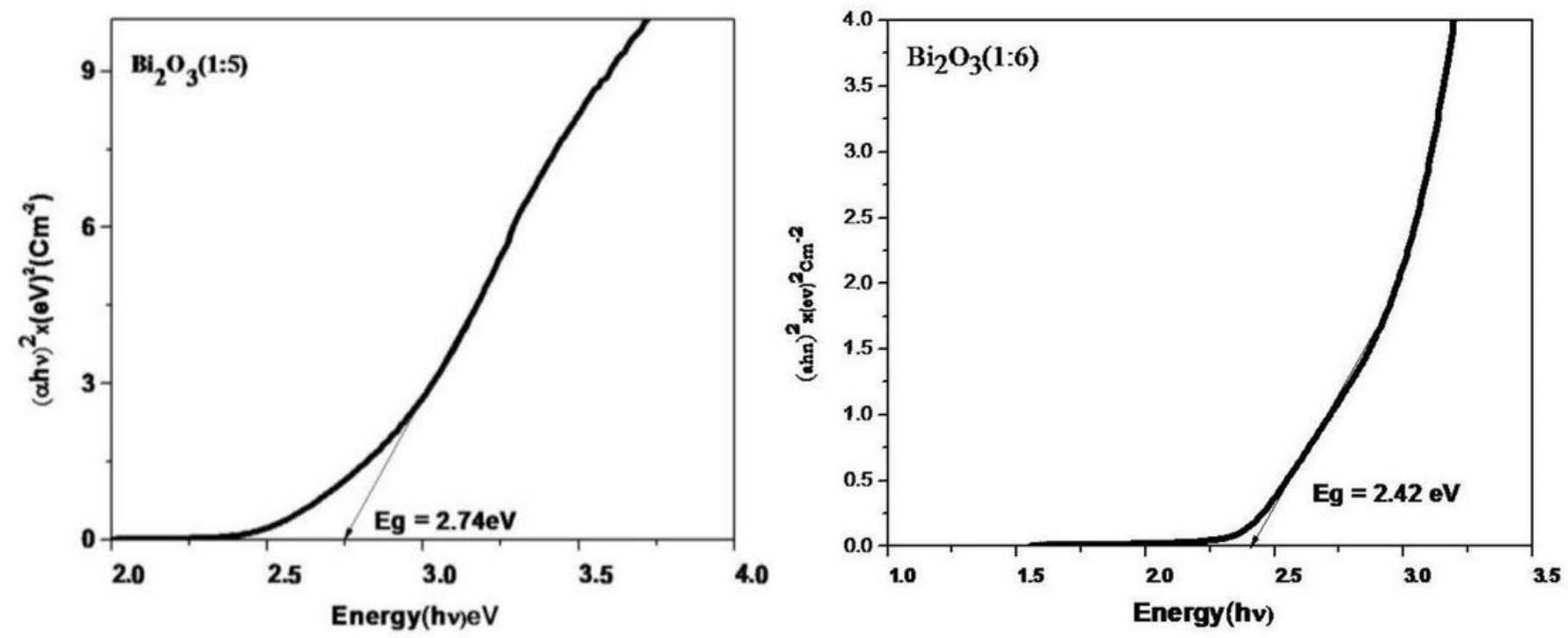

Figure 6

band gap analysis of $\mathrm{Bi} 2 \mathrm{O} 3$ (1:5) and (1:6) 


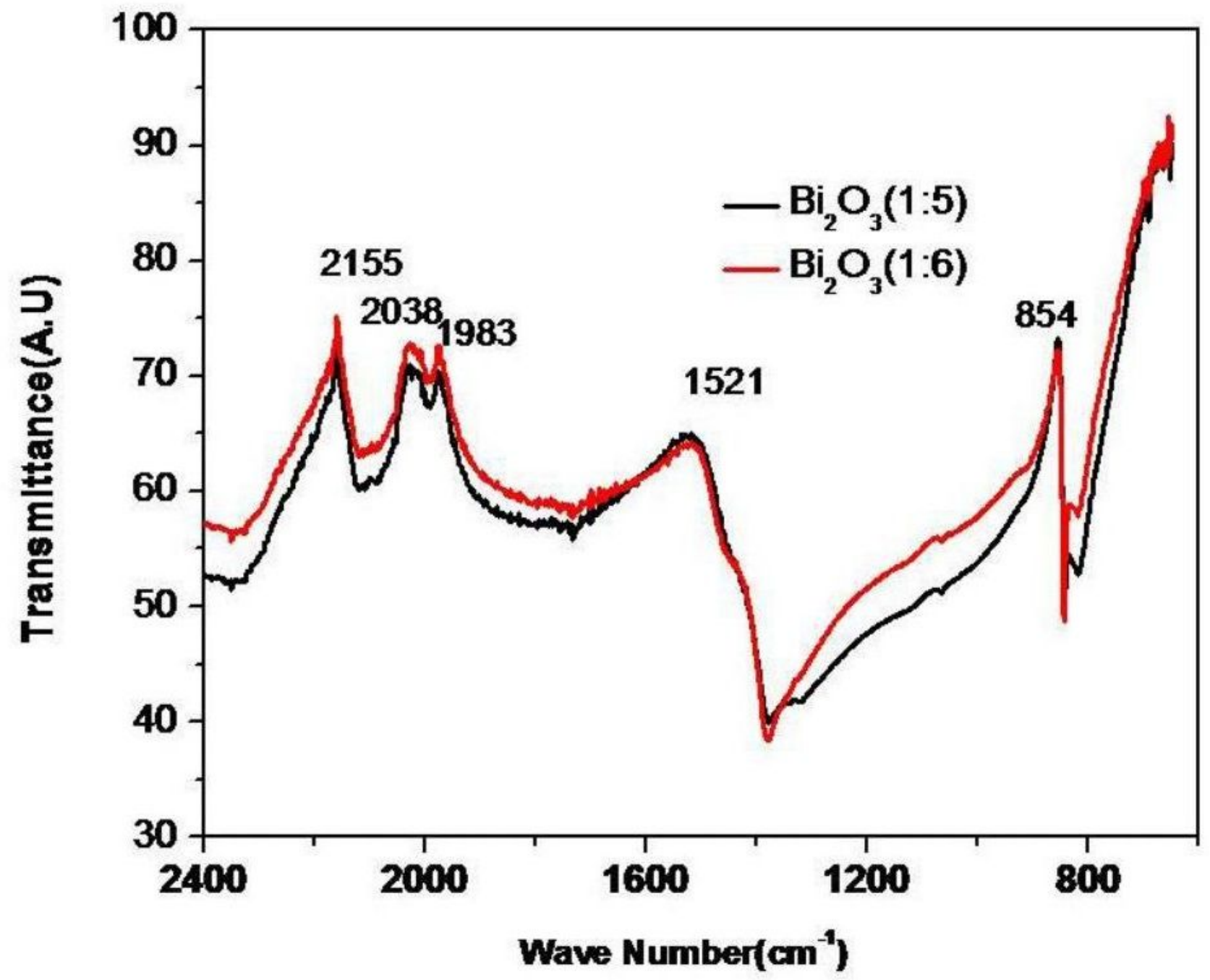

Figure 7

FTIR Analysis and its band gap of Bi2O3 (1:5)and(1:6)
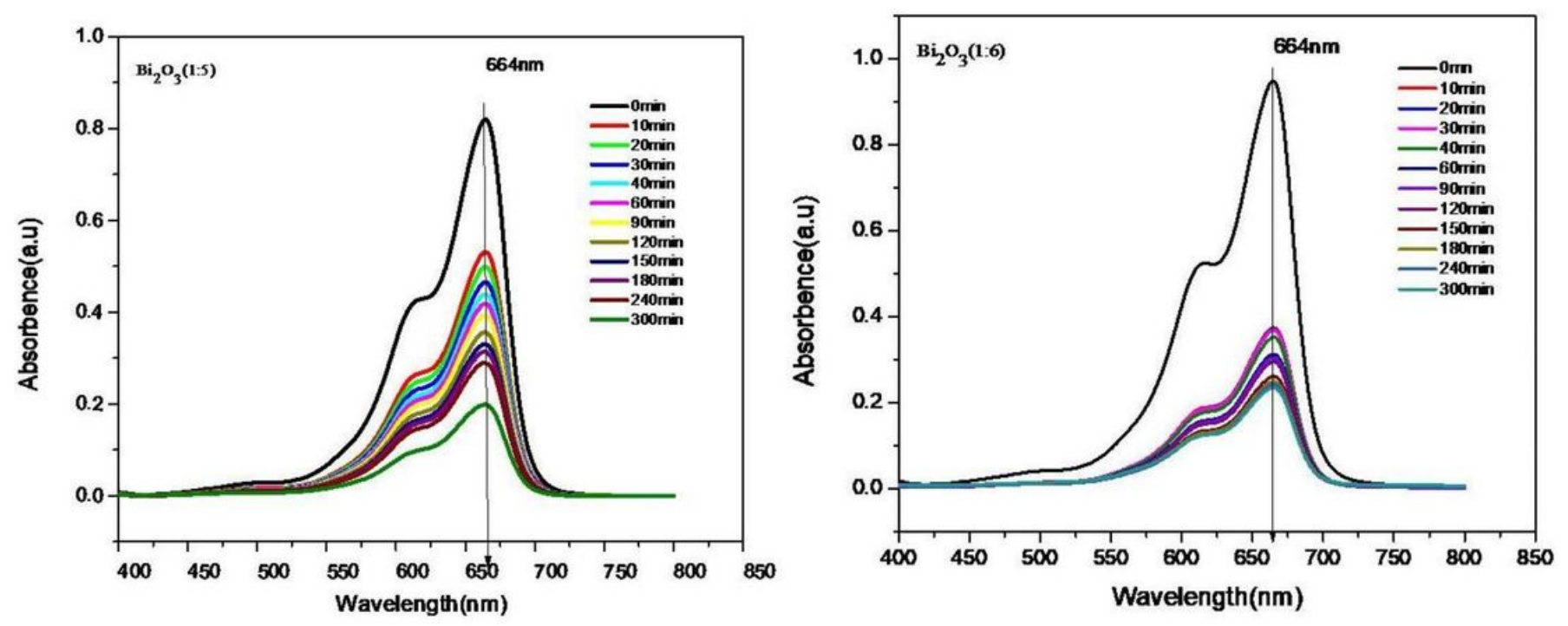


\section{Figure 8}

Time resolved UV-Vis spectra of MB solution in the presence of Bi203NPs (a) Bi2O3NPs (1:5) and (b) Bi203NPs (1:6)

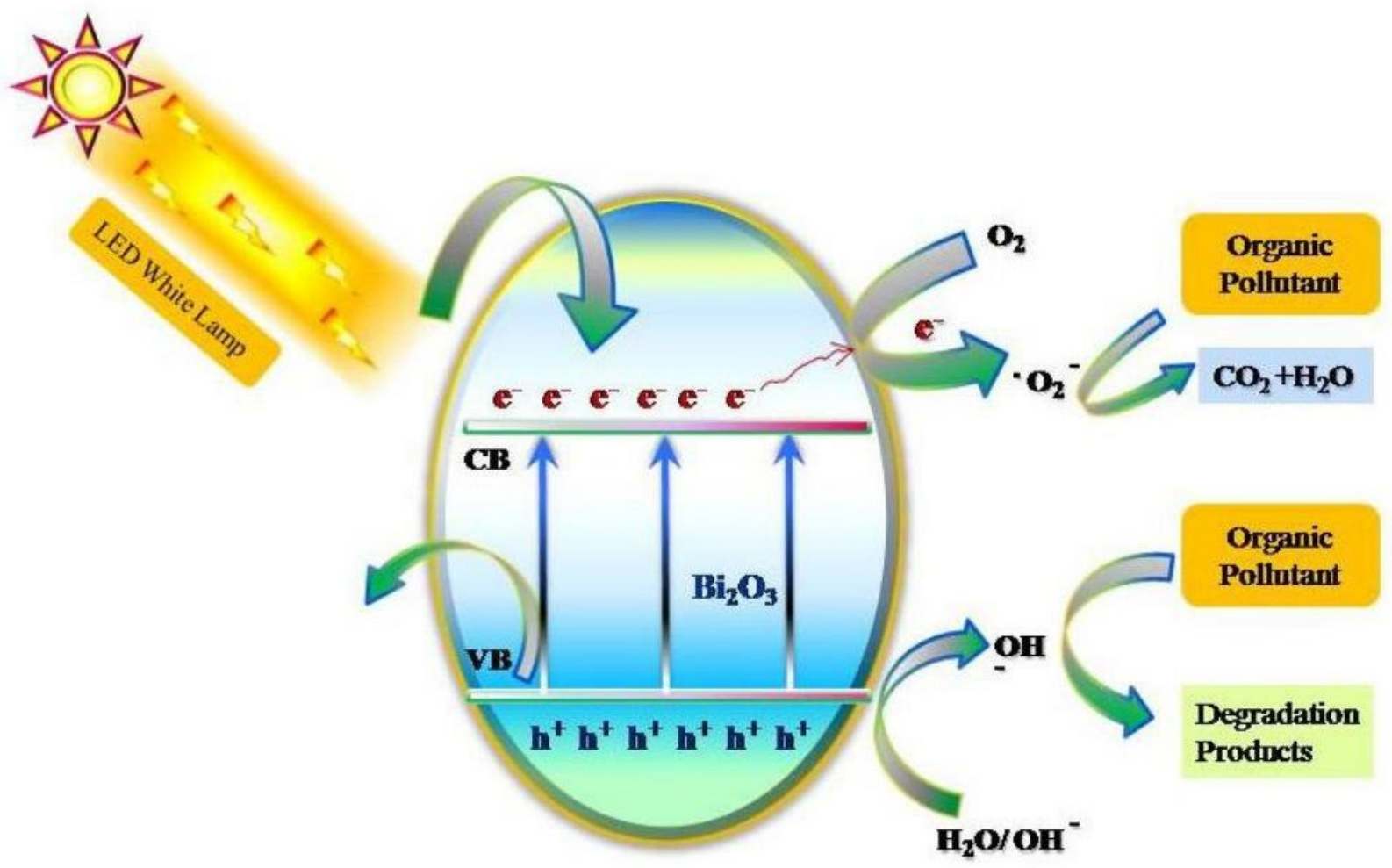

\section{Figure 9}

Schematic illustration of the possible photocatalytic reaction mechanism over Bi203 NPs 


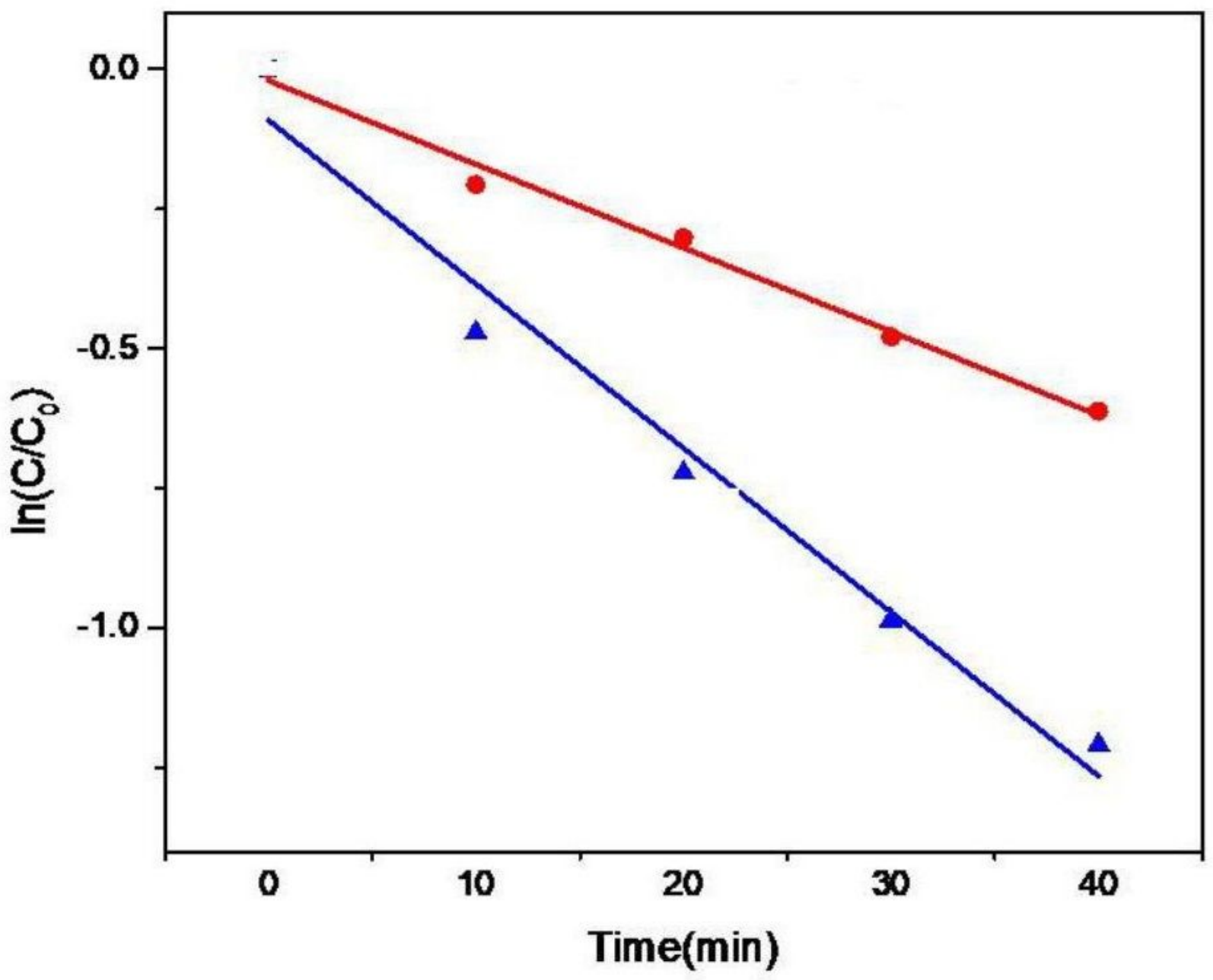

Figure 10

Photo degradation of MB dye in the presence of Bi203 NPs of (1:5) and (1:6) 